\title{
Understanding nonparametric estimation for clustered data
}

\author{
BY RICHARD HUGGINS \\ Centre for Mathematics and its Applications, Australian National University, Canberra, \\ ACT 0200, Australia \\ richard.huggins@anu.edu.au
}

\section{SUMMARY}

In this note we give an alternative formulation of the nonparametric estimators of Wang (2003) with the identity link. This results in a closed form of the estimator that has computational advantages and gives insight into the rationale behind the estimator.

Some key words: Clustered data; Nonparametric estimation.

\section{Introduction}

The development of nonparametric estimators for clustered data has had a relatively long history with limited success until the recent estimator of Wang (2003). The generalised estimating equation approach was examined by Lin \& Carroll (2000), who developed optimal estimating equations within a class of estimating equations, and showed that within the class considered the working independence estimator that ignores the correlation within the clusters is the most asymptotically efficient. Ruckstuhl et al. (2000) have examined several approaches and left as an open problem the possibility of obtaining an estimator with uniformly lower mean squared error than that of the working independence estimator. Wang (2003) developed estimating equations for the joint response in contrast to the conditional approach of Huggins (2004) and developed a full theory of her estimator. She showed in simulations and analytically that this estimator was superior to the working independence estimator, but it is not immediately apparent why it should be a good estimator. Here we determine a closed form of the estimator of Wang (2003) when the identity link is used.

\section{Estimating EQuATions}

Let $X^{\mathrm{T}}$ denote the transpose of the matrix $X$ and let $X^{-\mathrm{T}}=\left(X^{\mathrm{T}}\right)^{-1}$. To develop the estimating equations, consider a single cluster $Y=\left(Y_{1}, \ldots, Y_{m}\right)^{\mathrm{T}}$. Suppose $Y$ has covariance matrix $\Omega$ and mean vector $\mu=\left(\mu\left(z_{1}\right), \ldots, \mu\left(z_{m}\right)\right)^{\mathrm{T}}$, which is a function of covariates $\left(z_{1}, \ldots, z_{m}\right)^{\mathrm{T}}$. Let $\mu_{j}$ and $\Omega_{j}$ respectively denote the mean vector and the positive definite covariance matrix of $Y(j)=$ $\left(Y_{1}, \ldots, Y_{j}\right)^{\mathrm{T}}$. We are interested in estimating $\mu\left(z_{0}\right)$ for known $\Omega$. Wang et al. (2005) and Linton et al. (2004) comment on the effects of estimation of $\Omega$. To develop the nonparametric estimators, let $g_{p}(u)=\left(1, u, u^{2}, \ldots, u^{p}\right)$ so that, for $z$ near $z_{0}, \mu(z) \bumpeq \sum_{k=0}^{p} b_{k}\left(z-z_{0}\right)^{k}=g_{p}\left(z-z_{0}\right)^{\mathrm{T}} b$, where $b=\left(b_{0}, b_{1}, \ldots, b_{p}\right)$. If $u$ is a vector $u=\left(u_{1}, \ldots, u_{n}\right)$, let the matrix $G_{p}(u)$ have $j$ th row $\left(1, u_{j}, \ldots, u_{j}^{p}\right)$ so that, under regularity conditions, $\mu(z) \bumpeq G_{p}\left(z-z_{0}\right) b$.

Let $\bar{\mu}(z)$ denote the estimated value of $\mu(z)$ arising from the working independence estimator, let $\bar{\mu}=\left(\bar{\mu}\left(z_{1}\right), \ldots, \bar{\mu}\left(z_{m}\right)\right)^{\mathrm{T}}$ and let $\bar{\mu}(j)$ have $j$ th value $g_{p}\left(z_{j}-z_{0}\right) b$ and, for $k \neq j$, have value $\bar{\mu}\left(z_{k}\right)$. Let $d_{j}$ be a vector of length $m$ with 1 in the jth position and 0 elsewhere. In the Wang (2003) estimator 
with identity link, the contribution of $Y$ to the estimating equations is

$$
\sum_{j=1}^{m} K\left(z_{j}-z_{0}\right) g_{p}\left(z_{j}-z_{0}\right)^{\mathrm{T}} d_{j}^{\mathrm{T}} \Omega^{-1}\{Y-\bar{\mu}(j)\} .
$$

Now let $A$ denote the lower triangular Cholesky decomposition of $\Omega$, so that $\Omega=A A^{\mathrm{T}}$, and similarly define $A_{j}$ so that $\Omega_{j}=A_{j} A_{j}^{\mathrm{T}}$. Define standardised residuals by $Z_{j}=\left(Z_{1}, \ldots, Z_{j}\right)^{\mathrm{T}}=A_{j}^{-1}\left\{Y(j)-\mu_{j}\right\}$.

Write

$$
\Omega_{j+1}=\left[\begin{array}{cc}
\Omega_{j} & a_{j+1} \\
a_{j+1}^{\mathrm{T}} & b_{j+1}
\end{array}\right]
$$

and let $\ell_{j+1}^{\mathrm{T}}=a_{j+1}^{\mathrm{T}} A_{j}^{-\mathrm{T}}, \delta_{j+1}=\left(b_{j+1}-\ell_{j+1} \ell_{j+1}^{\mathrm{T}}\right)^{1 / 2}$ and $r_{j+1}^{\mathrm{T}}=-\delta_{j+1}^{-1} a_{j+1}^{\mathrm{T}} \Omega_{j}^{-1}$. Then, following Stewart (1973, p. 141) we have

Hence

$$
A_{j+1}=\left[\begin{array}{cc}
A_{j} & 0 \\
\ell_{j+1}^{\mathrm{T}} & \delta_{j+1}
\end{array}\right], \quad A_{j+1}^{-1}=\left[\begin{array}{cc}
A_{j}^{-1} & 0 \\
r_{j+1}^{\mathrm{T}} & \delta_{j+1}^{-1}
\end{array}\right] .
$$

$$
Z_{j+1}=r_{j+1}^{\mathrm{T}}\left\{Y(j)-\mu_{j}\right\}+\delta_{j+1}^{-1}\left(Y_{j+1}-\mu\left(z_{j+1}\right)\right)=\delta_{j+1}^{-1}\left[Y_{j+1}-\mu\left(z_{j+1}\right)-a_{j+1}^{\mathrm{T}} \Omega_{j}^{-1}\left\{Y(j)-\mu_{j}\right\}\right] .
$$

Let $\tilde{Y}_{j+1}=Y_{j+1}-a_{j+1}^{\mathrm{T}} \Omega_{j}^{-1}\left\{Y(j)-\mu_{j}\right\}$, so that, as $Z_{j+1}=\delta_{j+1}^{-1}\left(\tilde{Y}_{j+1}-\mu\left(z_{j+1}\right)\right)$, the pseudoobservations $\widetilde{Y}_{j}$ have means $\mu\left(z_{j}\right)$ and are uncorrelated.

Define $\bar{Z}(j)=A^{-1}\{Y-\bar{\mu}(j)\}$ and $\bar{Y}_{k}=Y_{k}-a_{k}^{\mathrm{T}} \Omega_{k-1}^{-1}\left\{Y(k-1)-\bar{\mu}_{k-1}\right\}$, where

$$
\bar{\mu}_{k-1}=\left(\bar{\mu}\left(z_{1}\right), \ldots, \bar{\mu}\left(z_{k-1}\right)\right)^{\mathrm{T}} .
$$

Note that $d_{j}^{\mathrm{T}} A^{-\mathrm{T}}$ has first nonzero element $\delta_{j}^{-1}$ and that the remaining terms are the $j$ th elements of $r_{k}(k>j)$. We denote these elements by $r_{k j}$ and let $\mathscr{R}$ be the matrix with rows $\mathscr{R}_{j}=\left(0, \ldots, 0, r_{j+1, j}, \ldots, r_{m, j}\right)$. Then (1) is

$$
\begin{aligned}
\sum_{j=1}^{m} K\left(z_{j}-z_{0}\right) g_{p}\left(z_{j}-z_{0}\right)^{\mathrm{T}} d_{j}^{\mathrm{T}} A^{-\mathrm{T}} A^{-1}\{Y-\bar{\mu}(j)\}= & \sum_{j=1}^{m} g_{p}\left(z_{j}-z_{0}\right)^{\mathrm{T}} K\left(z_{j}-z_{0}\right) \delta_{j}^{-1} \bar{Z}_{j}(j) \\
& +\sum_{j=1}^{m-1} g_{p}\left(z_{j}-z_{0}\right)^{\mathrm{T}} K\left(z_{j}-z_{0}\right) \sum_{k=j+1}^{m} r_{k j} \bar{Z}_{k}(j) \\
= & G_{p}\left(z-z_{0}\right)^{\mathrm{T}} W\left\{\bar{Y}-G_{p}\left(z-z_{0}\right) b\right\}+G_{p}\left(z-z_{0}\right)^{\mathrm{T}} K \bar{D} \\
& =G_{p}\left(z-z_{0}\right)^{\mathrm{T}} W\left\{\bar{Y}-G_{p}\left(z-z_{0}\right) b+\Delta^{2} \bar{D}\right\},
\end{aligned}
$$

where $W=K(z)^{1 / 2} R^{-1} K(z)^{1 / 2}$ for a diagonal weight matrix $K(z)$ and $R=\operatorname{diag}\left(\delta_{j}^{2}\right), \bar{D}$ has $j$ th element $\mathscr{R}_{j} \bar{Z}(j)$ and $\Delta$ is a diagonal matrix with $j$ th diagonal $\delta_{j}$.

The first term of (2) is related to estimating equations of Huggins (2004) and is easily interpreted. Simulations, not reported in detail, showed that estimation based on $G_{p}^{\mathrm{T}}\left(z-z_{0}\right) W\left\{\bar{Y}-G\left(z-z_{0}\right) b\right\}$ is far less efficient than that based on (2); in a small simulation study along the lines of that in Wang (2003), the ratios of the averages of the mean squared errors for the working independence estimator and the proposed estimators were 1.17 for the conditional estimator and 1.58 for Wang's estimators. Consider the second term in (2). Note that $b$ enters $\bar{Z}_{k}(j)$ for $k>j$ through $\bar{\mu}(j)$, where it is only involved in the $j$ th element. Let $\bar{\mu}_{k}(j)$ be the first $k$ elements of $\bar{\mu}(j)$ and let $\bar{Z}=A^{-1}(Y-\bar{\mu})$. Then, for $k>j$,

$$
\begin{aligned}
\bar{Z}_{k}(j) & =\delta_{k}^{-1}\left[Y_{k}-\bar{\mu}_{k}-a_{k}^{\mathrm{T}} \Omega_{k-1}^{-1}\left\{Y(k-1)-\bar{\mu}_{k-1}(j)\right\}\right] \\
& =\delta_{k}^{-1}\left(Y_{k}-\bar{\mu}_{k}\right)+r_{k}\left\{Y(k-1)-\bar{\mu}_{k-1}(j)\right\} \\
& =\delta_{k}^{-1}\left(Y_{k}-\bar{\mu}_{k}\right)+\sum_{\ell=1, \ell \neq j}^{k-1} r_{k \ell}\left(Y_{\ell}-\bar{\mu}_{\ell}\right)+r_{k j} Y_{j}-r_{k j} g_{p}\left(z_{j}-z_{0}\right) b \\
& =\bar{Z}_{k}+r_{k j}\left\{\bar{\mu}_{j}-g\left(z_{j}-z_{0}\right) b\right\}
\end{aligned}
$$


so that $\bar{Z}(j)=\bar{Z}+v_{j}\left\{\bar{\mu}-G_{p}\left(z-z_{0}\right) b\right\}$, where $v_{j}$ has $k$ th row consisting of zeros for $k \leqslant j$ and $v_{j k}=\left(0, \ldots, r_{k j}, \ldots, 0\right)$ for $k>j$. The contribution of a single cluster to the estimating equations from a single cluster may be expressed as

$$
G_{p}^{\mathrm{T}}\left(z-z_{0}\right) W\left\{\bar{Y}-G\left(z-z_{0}\right) b+\Delta^{2} \mathscr{R} \bar{Z}+\Delta^{2} S \bar{\mu}-\Delta^{2} T b\right\},
$$

where $S$ has $j$ th row $\mathscr{R}_{j} v_{j}$ and $T$ has $j$ th row $\mathscr{R}_{j} v_{j} G_{p}\left(z-z_{0}\right)$.

In practice we observe $n$ independent clusters and (3) yields the closed-form estimator,

$$
\hat{b}=\mathscr{A}_{1}^{-1} \sum_{i=1}^{n} G_{p}^{\mathrm{T}}\left(z_{i}-z_{0}\right) W_{i}\left(\bar{Y}_{i}+\Delta^{2} \mathscr{R} \bar{Z}_{i}+\Delta^{2} S \bar{\mu}_{i}\right)
$$

where $\mathscr{A}_{1}=\sum_{i=1}^{n} G_{p}^{\mathrm{T}}\left(z_{i}-z_{0}\right) W_{i}\left\{G_{p}\left(z_{i}-z_{0}\right)+\Delta^{2} T_{i}\right\}$ and $W_{i}$ and so on have the obvious definitions for the $i$ th cluster. As (3) is a reformulation of the estimator of Wang (2003) its properties are as given in Wang (2003).

\section{Discussion}

Lin et al. (2004) also give a closed form of the Wang (2003) estimator, but using pseudoobservations that are constructed differently from our $\tilde{Y}_{j}$, and Linton et al. (2004) also consider an approach based on pseudo-observations that are different again. The common idea is to construct these pseudo-observations so that they have the same mean as $Y_{j}$, but a diagonal covariance matrix for each cluster.

At present little theory is available to aid the construction of optimal nonparametric estimators for dependent data. One can construct estimators and derive their properties, but there is no general theory comparable to that of maximum likelihood to provide guidance. Weighted martingale estimators have a role for first-order Markov processes, as in Huggins \& Yip (1999) for example. Here the underlying process is not Markovian and efficiency is increased by incorporating this in the estimating equations. The formation of the Wang (2003) estimator derived here makes this explicit. Expression (3) is based on conditional residuals, which contribute in two ways to the estimating equations. The first is directly through the mean of $\bar{Y}$. The second is from noting that the 'conditional offset' $a_{k}^{\mathrm{T}} \Omega_{k-1}^{-1}\left(Y_{k-1}-\bar{\mu}_{k-1}\right)$ applied to $Y_{k}$ is also a function of $b$ for $k>j$. The complicated form of the estimating equations comes from resolving these two roles and (3) is the sum of the residuals in these roles.

Apart from the insights into the estimator that are gained from our derivation, the closed form of the estimator obviates the need for iterative numerical procedures to calculate the estimates. Typically the estimation of $\mu(z)$ requires one to compute several hundred estimates. If it is also necessary to estimate parameters in a model for $\Omega$ and use crossvalidation the computations quickly become unmanageable. The form of the estimator suggests several possible improvements. However, in simulations these improvements were not large. The standardised residuals employed here have previously been used in Huggins (1996) to develop robust estimators for clustered data.

\section{ACKNOWLEDGEMENT}

The author is grateful to Professor Alan Welsh for restimulating his interest in this problem and an anonymous referee for helpful comments.

\section{REFERENCES}

HugGins, R. M. (1996). Robust inference for variance components models for single trees of cell lineage data. Ann. Statist. 24, 1145-60.

Huggins, R. M. (2004). A note on nonparametric estimation for clustered data. Statist. Prob. Lett. 69, 129-33. HugGins, R. M. \& YIP, P. (1999). Estimation of the size of an open population from capture-recapture using weighted martingale methods. Biometrics 55, 387-95. 
Lin, X. \& Carroll, R. J. (2000). Nonparametric function estimation for clustered data when the predictor is measured with/without error. J. Am. Statist. Assoc. 95, 520-34.

Lin, X., Wang, N., Welsh, A. H. \& Carroll, R. J. (2004). Equivalent kernels of smoothing splines in nonparametric regression for clustered/longitudinal data. Biometrika 91, 177-93.

Linton, O. B., Mammen, E., Lin, X. \& Carroll, R. J. (2004). Correlation and marginal longitudinal kernel nonparametric regression. In Proceedings of the Second Seattle Symposium in Biostatistics. Analysis of Correlated Data, Ed. D. Y. Lin and P. J. Heargerty. New York: Springer.

Ruckstuhl, A. F., Welsh, A. H. \& Carroll, R. J. (2000). Nonparametric function estimation of the relationship between two repeatedly measured variables. Statist. Sinica 10, 51-71.

Stewart, G. W. (1973). Introduction to Matrix Computations. New York: Academic Press.

WANG, N. (2003). Marginal nonparametric kernel regression accounting for within-subject correlation. Biometrika 90, 43-52.

Wang, N., CARroll, R. J. \& Lin, X. (2005). Efficient semiparametric marginal estimation for longitudinal/clustered data. J. Am. Statist. Assoc. 100, 147-57.

[Received February 2005. Revised October 2005] 